\title{
Fertilidade e contracepção em mulheres com câncer em tratamento quimioterápico
}

\author{
Fertility and contraception in women with cancer treatment undergoing chemotherapy \\ Fertilidad y anticoncepción en mujeres con cáncer en tratamiento quimioterápico
}

\author{
Stephanie da Silva ${ }^{1}$ (B) \\ Renata Boer ${ }^{2}$ (D) \\ Lóris Aparecida Prado da $\mathrm{Cruz}^{2}$ (1) \\ Thais de Oliveira Gozzo ${ }^{3}$ (C)
}

1. Universidade de São Paulo, Escola de Enfermagem de Ribeirão Preto. Ribeirão Preto, SP, Brasil

2. Universidade de São Paulo, Escola de Enfermagem de Ribeirão Preto, Programa de Pós-graduação Enfermagem em Saúde Pública. Ribeirão Preto, SP, Brasil.

3. Universidade de São Paulo, Escola de Enfermagem de Ribeirão Preto, Departamento Materno Infantil e Saúde Pública. Ribeirão Preto, SP, Brasil.
Autor correspondente:

Thais de Oliveira Gozzo.

E-mail: thaisog@eerp.usp.br.

Recebido em 24/03/2020.

Aprovado em 23/06/2020.

DOI:https://doi.org/10.1590/2177-9465-EAN-2019-0374

\section{RESUMO}

Objetivo: identificar em mulheres em idade reprodutiva, com câncer e durante o tratamento quimioterápico, as orientações sobre preservação de fertilidade e planejamento reprodutivo e conhecer as informações fornecidas pela equipe de saúde. Métodos: estudo descritivo, realizado com mulheres na pré-menopausa, com diagnóstico de câncer e em tratamento quimioterápico. A coleta de dados foi realizada com apoio de um instrumento com informações sociodemográficas, sobre o câncer e seus tratamentos, informações referentes a preservação de fertilidade e uso de métodos contraceptivos antes e após o diagnóstico do câncer A análise dos dados foi feita por meio de estatística descritiva. Resultados: a média de idade das 49 participantes foi de 38,2 anos (DP=6,1) e 79,6\% estavam em tratamento devido ao câncer de mama. Quanto as informações recebidas sobre a importância do planejamento reprodutivo, $77,6 \%$ das participantes referiram que foram orientadas e $59,2 \%$ receberam tais orientações da equipe médica. Entretanto, em relação ao aconselhamento sobre métodos para manter a fertilidade, apenas, 6,1\% das participantes foram orientadas. Conclusão e Implicações para a prática: Deve-se considerar a relevância do aconselhamento especializado e a da manutenção de tomada de decisões ativas da mulher sobre a preservação de sua fertilidade.

Palavras-chave: Neoplasias; Quimioterapia Combinada; Infertilidade; Planejamento Familiar

\section{ABSTRACT}

Objective: to identify in women of reproductive age, with cancer and during chemotherapy treatment, the guidelines on fertility preservation and reproductive planning and to know the information provided by the health team. Methods: descriptive study, conducted with premenopausal women diagnosed with cancer and undergoing chemotherapy. Data collection was performed with the support of an instrument with sociodemographic information about cancer and its treatments, information regarding the preservation of fertility and the use of contraceptive methods before and after cancer diagnosis. Data analysis was performed using descriptive statistics. Results: the average age of the 49 participants was 38.2 years ( $S D=6.1$ ) and $79.6 \%$ were being treated for breast cancer. Regarding the information received about the importance of reproductive planning, $77.6 \%$ of participants reported that they were oriented and $59.2 \%$ received such guidance from the medical team. However, regarding counseling on methods to maintain fertility, only $6.1 \%$ of participants were counseled. Conclusion and Implications for practice: consideration should be given to the importance of expert counseling and the maintenance of active decision making by women about preserving their fertility.

Keywords: Neoplasms; Combined Chemotherapy; Infertility; Family Planning

\section{RESUMEN}

Objetivo: identificar en mujeres en edad reproductiva, con cáncer y durante el tratamiento de quimioterapia, las orientaciones sobre preservación de la fertilidad y planificación reproductiva y conocer las informaciones proporcionadas por el equipo de salud. Métodos: estudio descriptivo, realizado con mujeres premenopáusicas diagnosticadas con cáncer y sometidas a quimioterapia. La recolección de datos se realizó con apoyo de un instrumento con información sociodemográfica sobre el cáncer y sus tratamientos, información sobre la preservación de la fertilidad y uso de métodos anticonceptivos antes y después del diagnóstico del cáncer. El análisis de los datos se realizó mediante estadística descriptiva. Resultados: la media de edad fue de 38,2 años $(\mathrm{DE}=6,1)$, y $79,6 \%$ estaban en tratamiento para el cáncer de mama. Cuanto a la información recibida sobre la importancia de la planificación reproductiva, $77,6 \%$ de los participantes informaron que estaban orientados y $59,2 \%$ recibió orientación del equipo médico. Sin embargo, con respecto al asesoramiento sobre métodos para mantener la fertilidad, solo $6,1 \%$ de las participantes recibieron asesoramiento. Conclusión e Implicaciones para la práctica: Debe tenerse en cuenta la importancia de la asesoría experta y el mantenimiento de una toma de decisiones activa por parte de las mujeres para preservar su fertilidad.

Palabras clave: Neoplasias; Quimioterapia Combinada; Infertilidad; Planificación Familiar. 


\section{INTRODUÇÃO}

Aproximadamente $13 \%$ de todos os tipos de neoplasias acometem pessoas consideradas jovens ou abaixo dos 50 anos. ${ }^{1}$ Ao se planejar o tratamento oncológico de mulheres jovens, deve-se considerar que, a fertilidade pode ser afetada diretamente pelo câncer, pois este pode interferir na fisiologia dos órgãos reprodutores e/ou pelos tratamentos locais e sistêmicos. A quimioterapia, um dos tratamentos sistêmicos mais utilizados, têm impacto significante na fertilidade, sendo que drogas distintas apresentam diferentes graus de toxicidade, que variam de nenhum efeito à amenorreia transitória ou falência ovariana permanente e infertilidade. ${ }^{2}$ Ressalta-se que a infertilidade pode impactar de forma negativa nos planos reprodutivos da mulher e/ou casal que ainda não têm prole constituída. ${ }^{3}$

As preocupações com a fertilidade e o planejamento reprodutivo são questões-chave na atuação com esse grupo de pacientes. Entretanto, são consideradas ações distintas, pois enquanto a preservação da fertilidade está concentrada na toxicidade gonadotrófica causada pelos agentes quimioterápicos, o aconselhamento reprodutivo reconhece o potencial da ocorrência de uma gestação durante a quimioterapia, em especial, as não planejadas, e está focado na importância de se evitá-la. ${ }^{4}$

Com isso, a oncofertilidade surge no auxílio de mesclar conhecimentos da endocrinologia reprodutiva com a oncologia. ${ }^{5}$ O momento ideal para o encaminhamento das mulheres e esse tipo de serviço é um curto período de tempo, entre o diagnóstico e o início do tratamento. ${ }^{6} \mathrm{Na}$ atualidade, preservar o potencial de fertilidade, orientar e estimular o emprego de métodos contraceptivos, se tornou essencial no cuidado de mulheres jovens que são submetidas ao tratamento oncológico, ${ }^{7}$ em especial a quimioterapia, e fundamental no tratamento moderno do câncer.

É pertinente ressaltar que, os indivíduos em tratamento oncológico, sentem necessidade de que os profissionais da saúde, em especial, os médicos os apoiem quanto as preocupações referentes à fertilidade após o câncer. Dados observados em uma revisão sistemática sobre a necessidade de apoio à oncofertilidade para pacientes com câncer em idade reprodutiva ( 14 a 45 anos), constatou que estes desejam receber informações adequadas, ter acesso aos serviços de oncofertilidade, ter tempo para discutir o impacto do tratamento na fertilidade e apoio psicológico especializado. ${ }^{7}$

Tal preocupação se justifica quando se observa estudo ${ }^{8}$ que teve como objetivo identificar entre 630 mulheres jovens com câncer de mama, o quanto as preocupações referentes à fertilidade afetam as decisões acerca dos tratamentos contra a doença. Os resultados indicaram que, 37\% das participantes desejavam ter filhos no futuro, $51 \%$ se preocupavam com o risco de infertilidade e $26 \%$ disseram que este risco foi determinante em relação ao tratamento.

Deve-se considerar também que a preocupação com a infertilidade secundária à quimioterapia, associa-se à diminuição do bem-estar psicológico e aceitação da doença, impactando de forma negativa na qualidade de vida de indivíduos sobreviventes de câncer, com destaque para aqueles sem filhos. Ademais, ainda é possível identificar que a ameaça à fertilidade pode causar sentimento de perda e luto, e conflitos no relacionamento conjugal. $^{9}$

Em contrapartida, resultados de estudo realizado com mulheres com neoplasias apontaram que, apesar de não terem um diagnóstico clínico de infertilidade, muitas acreditavam que eram inférteis e, devido a essa crença, não usaram contracepção durante o tratamento, o que levou à gravidez não planejada. ${ }^{10}$ Outra pesquisa realizada com mulheres com câncer revelou que $55 \%$ acreditavam que não poderiam engravidar após o tratamento oncológico e, nessa perspectiva, $45 \%$ relataram que não utilizavam nenhum método contraceptivo. ${ }^{11}$

Apesar dos avanços na preservação da fertilidade e na disponibilidade de métodos contraceptivos para indivíduos com câncer, ainda se observa a necessidade de informações acerca dessas questões para as mulheres em idade reprodutiva. Diante disso, este estudo teve como objetivos identificar, em mulheres em idade reprodutiva, com câncer e durante o tratamento quimioterápico, as orientações sobre preservação de fertilidade e planejamento reprodutivo e conhecer as informações fornecidas pela equipe de saúde.

\section{MÉTODOS}

Estudo descritivo, desenvolvido em um hospital universitário no interior do estado de São Paulo com mulheres com diagnóstico de câncer durante tratamento quimioterápico. A coleta de dados ocorreu no período de janeiro a dezembro de 2018.

Adotou-se como critérios de inclusão mulheres com idade entre 18 e 45 anos, na pré-menopausa e que estivessem em tratamento quimioterápico durante o período de coleta de dados. Foram excluídas mulheres que estavam no ciclo gravídico-puerperal no momento do tratamento quimioterápico e/ou diagnóstico de metástase.

Para classificar o status menopausal utilizou-se os critérios propostos pelo Breast Cancer Surveillance Consortium (BCSC). O BCSC foi estabelecido em 1994, com o objetivo de aprimorar a compreensão das práticas de triagem do câncer de mama nos Estados Unidos da América. Para identificar o status menopausal, as participantes devem preencher um ou mais dos critérios apresentados no Quadro 1.12

As mulheres elegíveis responderam um questionário que continha informações sociodemográficas, tipo de tumor, tratamentos oncológicos realizados, história obstétrica e informações referente a preservação de fertilidade e uso de métodos contraceptivos antes e após o diagnóstico do câncer.

Os dados foram armazenados em planilha do Excel $2010^{\circledR}$ com dupla digitação e analisados pelo IBM SPSS 20 (Statistical Package for the Social Sciences). Foram realizadas análises descritivas, de tendência central e dispersão para as variáveis numéricas e frequência simples para as variáveis absolutas e relativas.

O estudo foi aprovado pelo Comitê de Ética em Pesquisa (Protocolo CAAE: 72423417.3.0000.5393), segundo as diretrizes e normas regulamentadoras de pesquisas envolvendo seres 
Quadro 1. Critérios para classificação do Status Menopausal segundo Breast Cancer Surveillance Consortium. ${ }^{12}$

\begin{tabular}{|l|l|}
\hline \multicolumn{3}{|c|}{ Classificação do Status Menopausal } \\
\hline Pré-menopausa & $\begin{array}{l}\text { - Períodos menstruais não pararam } \\
\text { - Uso atual de contraceptivos hormonais } \\
\text { - Menos de } 180 \text { dias desde o último período menstrual }\end{array}$ \\
\hline Perimenopausa & $\begin{array}{l}\text { - Não tem certeza se os períodos menstruais pararam } \\
\text { - De } 180 \text { a } 364 \text { dias desde o último período menstrual }\end{array}$ \\
\hline Pós-menopausa & $\begin{array}{l}\text { - Idade } \geq 55 \text { anos } \\
\text { - Relato de menopausa natural } \\
\text { - Ooforectomia bilateral }\end{array}$ \\
\hline Menopausa cirúrgica/outras & $\begin{array}{l}\text { - Uso atual de terapia de reposição hormonal } \\
\text { - 365 dias ou mais desde o último período menstrual }\end{array}$ \\
\hline
\end{tabular}

humanos, contidas na Resolução do Conselho Nacional de Saúde 466/2012.

\section{RESULTADOS}

Foram incluídas 49 mulheres, cuja idade variou de 24 a 45 anos, com média de idade de 38,2 anos ( $\mathrm{DP}=6,1)$, houve predomínio $(48,9 \%)$ da faixa etária de 40 aos 45 anos. A maioria das participantes referiu ter companheiro $(75,5 \%)$, frequentaram a escola por um período de nove 12 anos $(46,9 \%)$ e no momento da entrevista estavam de licença saúde (46,9\%), (Tabela 1).

Em relação ao histórico obstétrico das participantes, 89,8\% referiram que já engravidaram em algum momento de seu ciclo vital e 16,3\% apresentam a intenção de engravidar pela primeira vez ou ter mais filhos. Entre as participantes que já engravidaram, 31,8\% tiveram duas gestações e 6,8\% tiveram um aborto, (Tabela 2).

Quanto a localização do tumor, houve predominância de participantes com neoplasia de mama $(79,6 \%)$, seguida de colo de útero $(4,1 \%)$, ovário ( $2 \%$ ) e outras localidades $(14,3 \%)$. A modalidade terapêutica mais empregada, além da quimioterapia, foi o procedimento cirúrgico $(55,1 \%)$, a radioterapia $(12,2 \%)$ e a hormonioterapia (8,2\%).

Das participantes $57,1 \%$ referiram uso de algum método contraceptivo antes do diagnóstico de câncer, e após o diagnóstico $67,3 \%$. Observou-se alteração do método utilizado, sendo que antes do diagnóstico, a pílula anticoncepcional era o mais utilizado $(32,7 \%)$ e após o diagnóstico da doença o dispositivo intrauterino (DIU) passou a ser o método de escolha (42,9\%), (Tabela 3).

Com referência às informações recebidas sobre a importância do planejamento reprodutivo, $77,6 \%$ das participantes referiram que foram orientadas e 59,2\% receberam tais orientações da equipe médica. Entretanto, em relação ao aconselhamento sobre a manutenção da fertilidade, apenas três participantes referiram ter recebido informações (Tabela 4).

Das participantes que foram orientadas sobre a manutenção da fertilidade, apontaram que os métodos indicados foram o congelamento de óvulos $(66,6 \%)$ e o uso do fármaco acetato
Tabela 1. Distribuição das mulheres segundo a idade, estado marital, anos de estudo e ocupação $(n=49)$. Ribeirão Preto, São Paulo, Brasil, 2018.

\begin{tabular}{|lcc}
\hline \multicolumn{1}{c}{ Variáveis } & Freq. Absoluta & $\%$ \\
\hline Idade & & \\
\hline $24-29$ anos & 6 & $12,2 \%$ \\
\hline $30-34$ anos & 5 & $10,2 \%$ \\
\hline $35-39$ anos & 14 & $28,5 \%$ \\
\hline $40-45$ anos & 24 & $48,9 \%$ \\
\hline Estado marital & & \\
\hline Com companheiro & 37 & $75,5 \%$ \\
\hline Sem companheiro & 12 & $24,4 \%$ \\
\hline Anos de estudo & & \\
\hline Não frequentou & 1 & $2 \%$ \\
\hline Até quatro anos & 8 & $16,3 \%$ \\
\hline De cinco a oito anos & 10 & $20,4 \%$ \\
\hline De nove a 12 anos & 23 & $46,9 \%$ \\
\hline Acima de 13 anos & 7 & $14,3 \%$ \\
\hline Ocupação & & \\
\hline Do lar & 15 & $30,6 \%$ \\
\hline Licença saúde & 23 & $46,9 \%$ \\
\hline Autônoma & $11,4 \%$ \\
\hline Fon Banco & & \\
\hline
\end{tabular}

Fonte: Banco de dados do estudo

de gosserrelina (33,3\%). Contudo, apenas uma participante aceitou o recurso proposto, pela possibilidade de retomar seus planos de maternidade após a conclusão do tratamento. As outras duas participantes recusaram a intervenção pelo alto custo financeiro da medicação e pela possibilidade de atrasar o tratamento oncológico.

Além disso, 100\% das participantes apontam a importância de receber tais informações antes de iniciar a terapêutica 
oncológica, pois aumentam sua autonomia e podem direcioná-las nas tomadas de decisões. Dentre as respostas sobre os motivos de apontarem essas informações como relevantes, encontrou-se como explicação que essas informações lhes dão mais segurança na hora de priorizar o tratamento oncológico $(44,8 \%)$, as ajudam a compreender melhor a condição de saúde em que se encontram (32,7\%), evitam possíveis danos ao feto em caso de gravidez inesperada durante o tratamento

Tabela 2. Distribuição das mulheres segundo gravidez, número de gestações, número de abortos e intenção de ter mais filhos. Ribeirão Preto, São Paulo, Brasil, 2018.

\begin{tabular}{lcc}
\hline \multicolumn{1}{c}{ Variáveis } & Freq. absoluta & $\%$ \\
\hline Já engravidou em algum momento da vida & & $89,8 \%$ \\
\hline Sim & 44 & $10,2 \%$ \\
\hline Não & 5 & $22,7 \%$ \\
\hline Número de gestações & 10 & $31,8 \%$ \\
\hline 1 & 14 & $27,2 \%$ \\
\hline 2 & 12 & $6,8 \%$ \\
\hline 3 & 3 & $11,2 \%$ \\
\hline 4 & 5 & \\
\hline 5 ou mais & & $93,1 \%$ \\
\hline Número de abortos & 41 & $6,8 \%$ \\
Nenhum & 3 & $16,3 \%$ \\
\hline 1 & & $83,7 \%$ \\
\hline Intenção de engravidar pela 1a vez ou ter mais filhos & 8 & 41 \\
\hline Sim & & \\
\hline Não & & \\
\hline
\end{tabular}

Fonte: Banco de dados do estudo

Tabela 3. Distribuição das mulheres segundo o uso de métodos contraceptivos antes e após o diagnóstico de câncer. Ribeirão Preto, São Paulo, Brasil, 2018.

\begin{tabular}{lcc}
\hline \multicolumn{1}{c}{ Variáveis } & Freq. absoluta & $\%$ \\
\hline Uso de métodos contraceptivos antes do diagnóstico & & \\
\hline Sim & 28 & $57,1 \%$ \\
\hline Não & 21 & $42,9 \%$ \\
\hline Método utilizado & 16 & $32,7 \%$ \\
\hline Pílula anticoncepcional & 7 & $14,3 \%$ \\
\hline Injeção anticoncepcional & 3 & $6,1 \%$ \\
\hline Camisinha masculina & 2 & $4,1 \%$ \\
\hline Dispositivo intrauterino (DIU) & & \\
\hline Uso de métodos contraceptivos após o diagnóstico & 33 & $67,3 \%$ \\
\hline Sim & 16 & $32,6 \%$ \\
\hline Não & & \\
\hline Método utilizado & 21 & $42,9 \%$ \\
\hline Dispositivo intrauterino (DIU) & 10 & $20,4 \%$ \\
\hline Camisinha masculina & 1 & $2 \%$ \\
\hline Pílula anticoncepcional & 1 & $2 \%$ \\
\hline Injeção anticoncepcional & & \\
\hline
\end{tabular}

Fonte: Banco de dados do estudo 
Tabela 4. Distribuição das mulheres segundo as orientações recebidas quanto evitar gravidez durante o tratamento e aconselhamento sobre métodos para manter a fertilidade. Ribeirão Preto, São Paulo, Brasil, 2018.

\begin{tabular}{lcc}
\hline \multicolumn{1}{c}{ Variáveis } & Freq. absoluta \\
\hline $\begin{array}{l}\text { Recebeu orientação sobre a importância de não engravidar durante o tratamento } \\
\text { oncológico }\end{array}$ & 38 \\
\hline Sim & 11 & $77,6 \%$ \\
\hline Não & $22,4 \%$ \\
\hline Profissional que fez a orientação & 29 & 6 \\
\hline Médico & $59,2 \%$ \\
\hline Enfermeiro & 3 \\
\hline Médico e Enfermeiro & $12,2 \%$ \\
\hline Recebeu aconselhamento sobre manter a fertilidade & $6,1 \%$ \\
\hline Sim & 3 & $6,1 \%$ \\
\hline Não & 46 & $93,9 \%$ \\
\hline
\end{tabular}

Fonte: Banco de dados do estudo

$(18,4 \%)$ e a possibilidade de manter a fertilidade foi apontada por $4,1 \%$ das participantes.

\section{DISCUSSÃO}

No presente estudo, as participantes apresentaram idade entre 24 e 45 anos, em tratamento quimioterápico por diferentes tipos de câncer, 83,7\% relataram que não desejavam ter mais filhos, entretanto 32,6\% não faziam uso de método contraceptivo, estando em risco de gravidez não planejada. Dados corroborados por estudo ${ }^{13}$ que questionou 175 mulheres com neoplasia de mama sobre o uso atual de contraceptivo e sua intenção de engravidar futuramente, e $64 \%$ relataram que não utilizavam nenhum método no momento da pesquisa, apesar de não terem a intenção de engravidar. Resultados também observados em estudo ${ }^{10}$ que analisou o uso de contraceptivos durante o tratamento do câncer em um grupo de 107 mulheres em idade reprodutiva, e que encontrou que 40 delas estavam sexualmente ativas durante o tratamento e, destas, seis não utilizam nenhum método de contracepção.

Deve-se considerar que todas as mulheres sexualmente ativas, em idade reprodutiva, apresentam risco de gravidez não planejada na ausência de contracepção. Isso inclui mulheres com diagnóstico de câncer atual ou anterior. A gravidez não planejada no contexto de tratamento ou vigilância do câncer é uma questão delicada e pode acarretar dilemas clínicos e éticos para a mulher, parceiro, família e equipe de saúde. ${ }^{14}$

Quando comparadas as taxas de contracepção entre sobreviventes de câncer em idade reprodutiva e mulheres na população geral, as taxas ajustadas por idade e o uso de métodos contraceptivos foram menores entre as sobreviventes do que na população geral $(34 \%[28,8-40,0]$ em comparação com $53 \%$ [51,5-54,5], $\mathrm{P}<0,01)$. Além disso, apenas $56 \%$ das sobreviventes relataram ter recebido algum tipo de atendimento ou orientação referente ao planejamento reprodutivo (aconselhamento, prescrição ou procedimento relacionado ao controle da fertilidade) desde o diagnóstico oncológico. ${ }^{15}$

Tais dados corroboram com o observado no presente estudo, onde $22,4 \%$ das participantes apontaram que nenhum profissional da equipe de saúde orientou sobre a importância da utilização de métodos contraceptivos durante o tratamento oncológico. Dado semelhante, foi observado em estudo realizado com mulheres com câncer procedentes da cidade do México, onde o aconselhamento contraceptivo durante a quimioterapia, também foi precário. ${ }^{16}$

Observa-se uma dificuldade da equipe de saúde em abordar questões relacionadas ao planejamento reprodutivo, acarretando uma lacuna no cuidado à mulher jovem com diagnóstico de câncer. Sabe-se que o momento do diagnóstico é angustiante e sobrecarregado de informações, como apontado em revisão ${ }^{17}$ que discutiu aspectos relacionados a contracepção em sobreviventes de câncer em idade reprodutiva, e concluiu que orientações contraceptivas são cruciais durante a terapêutica, pois promovem segurança e autonomia à mulher, e fortalecem seu compromisso com o tratamento.

Observa-se que se as metas reprodutivas da mulher não forem abordadas especificamente, existe a possibilidade de ocorrer uma gravidez indesejada e de não preservar a fertilidade. ${ }^{18}$ Dessa forma, torna-se fundamental que todas as mulheres sejam informadas sobre o risco da diminuição ou da perda da fertilidade após a utilização de terapêutica oncológica.

Outro ponto de destaque a ser considerado durante o tratamento oncológico em mulheres em idade reprodutiva, é a preservação da fertilidade. Deve-se ressaltar que a manutenção da fertilidade durante o processo de doença envolve aspectos além do contexto físico da vida da mulher, uma vez que descrevem a infertilidade induzida pela quimioterapia como um sentimento de agonia, que põe em risco seu senso de autorrealização e identidade feminina. ${ }^{19}$ 
No presente estudo, foi observado que $16,3 \%$ das participantes apontaram o desejo de engravidar após o término do tratamento quimioterápico, contudo apenas $6,1 \%$ receberam aconselhamento para preservar a fertilidade. Dados corroborados por estudo americano com 211 mulheres com câncer de mama em tratamento quimioterápico, onde $52 \%$ não receberam aconselhamento sobre a preservação da fertilidade. ${ }^{4}$ Também observado em estudo desenvolvido no Líbano ${ }^{20} \mathrm{com} 39$ mulheres com câncer de mama e $76,9 \%$ não discutiram com o oncologista a possibilidade de infertilidade.

De acordo com as recomendações da American Society of Clinical Oncology (ASCO), ${ }^{21}$ os oncologistas devem se preparar para discutir infertilidade e o potencial risco das terapias, o mais breve possível a partir do diagnóstico do câncer, podendo ser simultâneo ao estadiamento e o planejamento do tratamento. Recomendam ainda, o encaminhamento das mulheres que manifestarem interesse na fertilidade, bem como aquelas que não tiverem certeza, para especialistas em reprodução o mais breve possível; além de referenciar para atendimento psicológico.

Apesar de diversas técnicas de preservação de fertilidade estarem disponíveis, o uso destas tem sido limitado por falta de aconselhamento sobre o assunto. ${ }^{17}$ Abordar a fertilidade e a sua preservação tem sido um tópico pouco explorado, como mostrou nos resultados de scoping review, ${ }^{22}$ destacando que as dificuldades e barreiras para o acesso a serviços especializados são semelhantes entre os países desenvolvidos e em desenvolvimento. Nesse sentido, nota-se que uma das implicações para a prática, é que as mulheres com câncer ou histórico de câncer precisam de acesso a serviços que ofereçam opções para planejamento reprodutivo e de preservação da fertilidade. ${ }^{22}$

Também foram consideradas barreiras para a preservação da fertilidade: falta de comunicação com os pacientes por parte da equipe de saúde, ${ }^{22}$ dificuldade para referenciar os pacientes para serviços especializados, ${ }^{6,22}$ nível de treinamento/conhecimento dos diferentes profissionais da saúde que atuam com essa população, ${ }^{6,22}$ hesitação dos pacientes quanto ao desejo de preservar a fertilidade ${ }^{22}$ e os custos. ${ }^{22}$

Quanto aos custos, estes variam de país para país, e são influenciados pelos aspectos legais, pela idade do paciente, pela cultura local, e a cobertura dos diferentes sistemas de saúde, como descrito no estudo ${ }^{6}$ desenvolvido em 40 centros de oncofertilidade de 28 países. Este estudo aponta ainda as estimativas de cobertura dos custos para os diferentes países, e refere que no Brasil os custos quanto a criopreservação de embrião, tecido ovariano e de esperma, são de responsabilidade do paciente e variam de região para região.

Além desses aspectos, destaca-se o aconselhamento especializado sobre a perda da função reprodutiva e preservação da fertilidade, como demostrou os resultados de estudo ${ }^{23}$ recente que buscou compreender a prática e as percepções, de profissionais médicos e enfermeiros, quanto às barreiras para a triagem e o encaminhamento de mulheres jovens para contracepção e preservação da fertilidade durante o tratamento do câncer. Os profissionais não tinham clareza de seus papéis e responsabilidades frente a temática e pressupunham que outro membro da equipe havia abordado a mulher. Concluindo que a equipe não possuía treinamento sobre contracepção e preservação da fertilidade, o que impedia discussões e aconselhamento apropriado e oportuno.

Dentre os profissionais da saúde atuantes com esse grupo de paciente, destaca-se o profissional enfermeiro navegador, que favorece que estes tenham informações para participar ativamente do processo de cuidado. Além de estabelecer a comunicação entre os diferentes profissionais da saúde envolvidos na assistência, facilitando a jornada do paciente oncológico. ${ }^{22}$

\section{CONCLUSÃO E IMPLICAÇÕES PARA A PRÁTICA}

Apesar da maioria das participantes do atual estudo receberem orientações acerca do uso de método contraceptivo para evitarem uma gestação não planejada durante o tratamento oncológico, observou-se que ainda existe falha nas informações sobre esse assunto. $\mathrm{O}$ mesmo pode ser observado referente aos métodos de preservação da fertilidade, o qual foi possível identificar que apesar de se tratar de uma temática relevante e atual, ainda não faz parte da rotina oferecer essas informações e nem meios de atingi-la. Deve-se considerar a relevância do aconselhamento especializado e a da manutenção de tomada de decisões ativas da mulher sobre seu planejamento reprodutivo.

Nesse sentido, destaca-se a figura do enfermeiro para atuar no aconselhamento para o uso de métodos contraceptivos e para preservação da fertilidade, uma vez que esse profissional é preparado para desempenhar suas funções nas diversas etapas do tratamento oncológico.

Pode-se apontar como limitações do estudo o número de participantes e a coleta de dados em um único serviço, porém esse hospital é referência para tratamento de infertilidade e disponibiliza atendimento para contracepção e preservação de fertilidade para pacientes com cancer.

\section{FINANCIAMENTO}

"O presente trabalho foi realizado com apoio da Coordenação de Aperfeiçoamento de Pessoal de Nível Superior - Brasil (CAPES) - Código de Financiamento 001", bolsa de mestrado concedida a Renata Boer e bolsa de doutorado concedida a Lóris Aparecida Prado da Cruz.

\section{CONTRIBUIÇÕES DOS AUTORES}

Desenho do estudo de revisão. Coleta e análise de dados. Interpretação dos resultados. Redação e revisão crítica do manuscrito. Aprovação da versão final do artigo. Responsabilidade por todos os aspectos do conteúdo e a integridade do artigo publicado. Thais de Oliveira Gozzo.

Coleta e análise de dados. Interpretação dos resultados. Redação e revisão crítica do manuscrito. Aprovação da versão final do artigo. Responsabilidade por todos os aspectos do 
conteúdo e a integridade do artigo publicado. Stephanie da Silva. Lóris Aparecida Prado da Cruz.

Análise de dados e interpretação dos resultados. Redação e revisão crítica do manuscrito. Aprovação da versão final do artigo. Responsabilidade por todos os aspectos do conteúdo e a integridade do artigo publicado. Renata Boer.

\section{EDITOR ASSOCIADO}

\section{Aline Aparecida Monroe}

\section{REFERÊNCIAS}

1. American Cancer Society. Cancer facts and figures 2017. Atlanta: American Cancer Society, 2017 [citado 2019 nov 14]. Disponível em: https://www.cancer.org/content/dam/cancer-org/research/cancer-factsand-statistics/annual-cancer-facts-and-figures/2017/cancer-facts-andfigures-2017.pdf

2. Jayasinghe $\mathrm{YL}$, Wallace WHB, Anderson RA. Ovarian function, fertility and reproductive lifespan in cancer patients. Expert Rev Endocrinol Metab. 2018;13(3):125-36. http://dx.doi.org/10.1080/17446651.2018 1455498. PMid:30058903.

3. Madrigal JM, Atluri M, Radeke EK, Patel A. Looking through the lens of a family planner to prioritize reproductive health among women with cancer. J Oncol Pract. 2019;15(2):e141-52. http://dx.doi.org/10.1200/ JOP.18.00429. PMid:30763204

4. Johansen SL, Lerma K, Shaw KA. Contraceptive counseling in reproductive-age women treated for breast cancer at a tertiary care institution: a retrospective analysis. Contraception. 2017;96(4):248-53. http://dx.doi.org/10.1016/j.contraception.2017.06.004. PMid:28645785.

5. Ashizawa M, KandaY. Preservation of fertility in patients with hematological malignancies. Jpn J Clin Oncol. 2020;50(7):729-42. http://dx.doi. org/10.1093/jjco/hyaa043. PMid:32419028.

6. Rashedi AS, Roo SF, Atamen LM, Edmonds ME, Silva AA, Scarella $A$ et al. Survey of fertility preservation options available to patients with cancer around the globe. JCO Glob Oncol. 2020;6:JGO.2016.008144. PMid:32259160.

7. Logan S, Perz J, Ussher JM, Peate M, Anazodo A. A systematic review of patient oncofertility support needs in reproductive cancer patients aged 14 to 45 years of age. Psychooncology. 2018;27(2):401-9. http:// dx.doi.org/10.1002/pon.4502. PMid:28734119.

8. Ruddy KJ, Gelber SI, Tamimi RM, Ginsburg ES, Schapira L, Come SE et al. Prospective study of fertility concerns and preservation strategies in young women with breast cancer. J Clin Oncol. 2014 abr 10;32(11):1151-6. http://dx.doi.org/10.1200/JCO.2013.52.8877. PMid:24567428.

9. Ussher JM, Perz J. Infertility-related distress following cancer for women and men: a mixed method study. Psychooncology. 2019;28(3):607-14 http://dx.doi.org/10.1002/pon.4990. PMid:30659694.

10. Maslow BS, Morse CB, Schanne A, Loren A, Domchek SM, Gracia CR. Contraceptive use and the role of contraceptive counseling in reproductiveaged women with cancer. Contraception. 2014 jul;90(1):79-85. http:// dx.doi.org/10.1016/j.contraception.2014.03.002. PMid:24792148.
11. Patel A, Sreedevi M, Malapati R, Sutaria R, Schoenhage MB, Patel AR et al. Reproductive health assessment for women with cancer: a pilot study. Am J Obstet Gynecol. 2009;201(2):191.e1-4. http://dx.doi. org/10.1016/j.ajog.2009.04.021. PMid:19539896.

12. Ballard-Barbash R, Taplin SH, Yankaskas BC, Ernster VL, Rosenberg RD, Carney PA et al. Breast Cancer Surveillance Consortium: a national mammography screening and outcomes database. AJR Am J Roentgenol 1997 out:169(4):1001-8. http://dx.doi.org/10.2214/ajr.169.4.9308451. PMid:9308451.

13. Kopeika J, Bhaduri M, Kugadas A, Reddy N, Shewbridge A, Mukherj $D$ et al. Planned and unplanned pregnancies in breast cancer survivor. Breast. 2019;46:75-80. http://dx.doi.org/10.1016/j.breast.2019.05.004. PMid:31100574.

14. Crafton SM, Lynch CD, Cohn DE, Eisenhauer EL. Reproductive counseling, contraception, and unplanned pregnancy in fertile women treated by gynecologic oncologists. Gynecol Oncol Rep. 2016;19:22-6. http://dx.doi.org/10.1016/j.gore.2016.11.006. PMid:28018956.

15. Dominick SA, McLean MR, Whitcomb BW, Gorman JR, Mersereau JE, Bouknight JM et al. Contraceptive practices among female cancer survivors of reproductive age. Obstet Gynecol. 2015;126(3):498-507. http://dx.doi.org/10.1097/AOG.0000000000000963. PMid:26181090.

16. Castro-Sanchez A, Martinez-Cannon BA, Platas A, Mohar A, Fonseca $A$, Vega $Y$ et al. Suboptimal use of effective contraceptive methods in young Mexican women with breast cancer. J Glob Oncol. 2018;4(4):1-7. http://dx.doi.org/10.1200/JGO.18.00064. PMid:30300053.

17. Shliakhtsitsava $K$, Suresh D, Hadnott T, Su IH. Best practices in counseling young female cancer survivors on reproductive health. Semin Reprod Med. 2017;35(4):378-89. http://dx.doi.org/10.1055/s-0037-1603770. PMid:29036745.

18. Crafton S, Nekkanti S, Lynch C, Cohn DE, Fowler JM, Copeland $\mathrm{LJ}$ et al. Documentation of pregnancy risk assessment and pregnancy among women presenting for gynaecologic oncology consultation Int J Gynecol Cancer. 2016;26(1):35-42. http://dx.doi.org/10.1097/ IGC.0000000000000576. PMid:26658364.

19. Dagan E, Modiano-Gattegno S, Birenbaum-Carmeli D. "My choice": breast cancer patients recollect doctors fertility preservation recommendations. Support Care Cancer. 2017;25(8):2421-8. http://dx.doi.org/10.1007/ s00520-017-3648-1. PMid:28238094.

20. Assi HI, Kakati RT, Attieh RM, Khoury J, Sukhon F, Berro J et al. Fertility in breast cancer survivors in the Middle East: a retrospective study. Breast. 2020;52:58-63. http://dx.doi.org/10.1016/j.breast.2020.04.010. PMid:32388348.

21. Oktay K, Harvey BE, Partridge AH, Quinn GP, Reinecke J, Taylor HS et al Fertility preservation in patients with cancer: ASCO clinical practice guideline update. J Clin Oncol. 2018;36(19):1994-2001. http://dx.doi. org/10.1200/JCO.2018.78.1914. PMid:29620997.

22. Anazodo A, Laws P, Logan S, Saunders C, Travaglia J, Gerstl B et al How cam we improve oncofertulity care for patients? A sistematic scoping review of current international practice and models of care. Hum Reprod Update. 2019;25(2):159-79. http://dx.doi.org/10.1093/ humupd/dmy038. PMid:30462263.

23. Lindsay SF, Woodhams EJ, White KO, Drainoni ML, Johnson NL, Yinusa-Nyahkoon L. Understanding barriers to contraception screening and referral in female adolescents and young adults with cancer. $J$ Adolesc Young Adult Oncol. 2020;9(1):63-71. http://dx.doi.org/10.1089/ jayao.2019.0074. PMid:31634022. 